Davis and H. H. Hosack, J. Appl. Phys. 34, 864 (1963); H. H. Hosack, ibid. 36, 1281 (1965); L. V. Iogansen, Zh. Eksperim. i Teor. Fiz. 45, 207 (1963); 47, 270 (1964) [Soviet Phys. JETP 18, 146 (1964); 20, 180 (1965)]; R. Hrach, Czech. J. Phys. 20B, 32 (1970); 20B, 307 (1970).

${ }^{7}$ A. Modinos and N. Nicolaou, Surface Sci. 17, 359 (1969).

${ }^{8}$ M. S. Child, Mol. Phys. 12, 401 (1967)。

${ }^{9}$ J. N. L. Connor, Mol. Phys. 15,37 (1968); 19,65 (1970).

${ }^{10}$ N. Fröman and Ö. Dammert, Nucl. Phys. 147A, 627 (1970).

${ }^{11} \mathrm{C}$. Y. Wong and J. Bang, Phys. Letters $\underline{29 \mathrm{~B}}, 143$ (1969); A. V. Ignatyuk, N. S. Rabotnov, and G. N. Smirenkin, ibid. 29B, 209 (1969); E. V. Gaĭ, A. V. Ignatyuk, N. S. Rabotnov, and G. N. Smirenkin, Yadern. Fiz. 10, 542 (1969) [Soviet J. Nucl. Phys. 10, 311 (1970)]; L. I. Ponomarev, Lectures on Quasiclassics ITF-67-53 (Institute for Theoretical Physics, Acad. Sci. Ukrain. SSSR, Kiev, 1968), quoted in Ref. 10; A. Skorupski (unpublished), quoted in Ref. 10; J. D. Cramer and J. R. Nix, Phys. Rev. C 2, 1048 (1970).

${ }^{12}$ Equation (1) has also been derived, in a similar way to that of Ref.9, in Ref. 7. Equation (1) also agrees with the first-order phase integral derivation of Fröman and Dammert (see Ref. 10).

${ }^{13}$ E. L. Murphy and R. H. Good, Phys. Rev. 102 , 1464 (1956); S. G. Christov, Phys. Status Solidi 17, 11 (1966); 21, 159 (1967).

${ }^{14}$ R. A. Buckingham and A. Dalgarno, Proc. Roy.
Soc. (London) 213A, 506 (1952); M. V. Berry, Proc. Phys. Soc. (London) 88, 285 (1966); P. M. Livingston, J. Chem. Phys. 45, 601 (1966).

${ }^{15}$ K. W. Ford, D. L. Hill, M. Wakano, and J. A. Wheeler, Ann. Phys. (N.Y.) 7, 239 (1959); R. M。 Eisberg and C. E. Porter, Rev. Mod. Phys. $\underline{33}, 190$ (1961); G. V. Dubrovsky, Opt. i Spektroskopiya 17, 771 (1964) [Opt. Spectry. USSR 17, 416 (1964)]; R。 R。 Herm, J. Chem. Phys. 47, 4290 (1967); W. H. Miller, ibid. 48, 1651 (1968); J. N. L. Connor, Mol. Phys. $\underline{15}, 621$ (1968); 16, 525 (1969); see also J. N. L. Connor and M. S. Child, ibid. 18, 653 (1970); J. N. L. Connor, Chem. Phys. Letters $\underline{4}, 419$ (1969); A。S. Dickinson, Mol. Phys。18, 441 (1970); N. Fröman and P. O. Fröman, Nucl. Phys。147A, 606 (1970).

${ }^{16} \mathrm{E}$. Guth and C. J. Miller, Phys. Rev. 59, 575 (1941); O. E. H. Rydbeck, J. Appl. Phys。13, 577 (1942); S。C. Miller and R. H. Good, Phys. Rev. $\underline{91}, 174$ (1953); D. L. Hill and J. C. Wheeler, ibid. $\underline{89}, 1102$ (1953); V. A. Fock, Electromagnetic Diffraction and Propagation Problems (Pergamon, Oxford, England, 1965), p. 308.

${ }^{17}$ M. Soop, Arkiv. Fisik 30, 217 (1965); N. I. Zhirnov, Izv. Vysshiky Uchelon. Zarederii Fiz. No. 4, 28 (1965); No. 5, 41 (1966); No. 6, 101 (1966); No. 6, 108 (1966) [Soviet Phys. J. No. 4, 16 (1965); $\underline{9}, 25$ (1966); $\underline{9}, 56$ (1966); 9 , 61 (1966)]; A. S. Dickinson, Ref. 15.

${ }^{18} \mathrm{~T}$. Y. Wu and T. Ohmura, Quantum Theory of Scattering (Prentice-Ha1l, Englewood Cliffs, N. J., 1962), Chap. 1, Sec. A.

\title{
Influence of Combined Static and Time-Dependent Quadrupole Interactions on Angular Correlation in Nuclei with Integral Spin*
}

\author{
P. da R. Andrade ${ }^{\dagger}$ and J. D. Rogers ${ }^{\dagger}$ \\ Instituto de Física, Universidade Federal do Rio Grande do Sul, Pôrto Alegre, Brasil
} (Received 22 September 1970)

\begin{abstract}
The application of the Bloch-Wangsness-Redfield theory of nuclear relaxation to the study of perturbed angular correlations in even- $A$ nuclei subject to simultaneous static and timedependent quadrupole interactions has been treated. The differences in the perturbation factors as compared to the odd- $A$ case are shown.
\end{abstract}

In a recent paper ${ }^{1}$ (denoted below by I), we have treated the application of the Bloch-WangsnessRedfield theory of nuclear relaxation to the study of perturbed angular correlations (PAC) in odd- $A$ nuclei subject to simultaneous static and time-dependent quadrupole interactions. Stimulated by recent experimental results ${ }^{2}$ we have investigated the extension of this formalism to the case of even$A$ nuclei (integral spins).

Problems may be expected in the case of integral spins due to the degeneracy of the hyperfine transitions $+\alpha \rightarrow 0$ and $-\alpha \rightarrow 0$, where $\alpha$ represents the spin projection $m_{j}$. The result of this degeneracy in NMR studies is that the resonance line corresponding to these transitions may have a shape made up of a superposition of Lorentzians, ${ }^{3}$ which would correspond to the appearance of a combination of exponentials for the relaxation of the associated frequency in the perturbed angular-correlation spectrum. It is the purpose of this addendum to show that this effect will in fact occur in perturbed angular-correlation spectrum.

To demonstrate this, we review first the solution of the odd- $A$ problem. The basic equation to be solved is (2.16) of $\mathrm{I}$ :

$$
\dot{\rho}_{\alpha \alpha^{\circ}}^{*}=\sum_{\beta \beta^{\prime}} R_{\alpha \alpha^{\prime} \beta \beta^{\prime}} \rho_{\beta \beta^{\prime}}^{*}, \quad \alpha-\alpha^{\prime}=\beta-\beta^{\prime}
$$

where the matrix elements $R_{\alpha \alpha^{\prime} \beta \beta^{\prime}}$ are combinations of various spectral densities of the form $\partial_{\alpha \alpha^{\prime} \beta \beta^{\prime}}(\omega)=\int_{-\infty}^{\infty}\left\langle\left(\alpha|K(t)| \alpha^{\prime}\right)\left(\beta^{\prime}|K(t-\tau)| \beta\right)\right\rangle_{e} e^{-i \omega \tau} d \tau$ 。 
Equation (1) can be separated into diagonal $\left(\alpha=\alpha^{\prime}\right.$, $\left.\beta=\beta^{\prime}\right)$ and off-diagonal parts which are uncoupled. In the solution of the off-diagonal parts it becomes necessary to evaluate matrix elements of the form $R_{\alpha \alpha^{*}-\alpha-\alpha^{*}}$ which depend on spectral densities of the form

$$
J_{\alpha \beta \alpha-\beta}(\omega)=\int_{-\infty}^{\infty}\langle(\alpha|K(t)| \beta)(-\beta|K(t+\tau)| \alpha)\rangle_{e} e^{-i \omega \tau} d \tau,
$$

$\oint_{\alpha \alpha^{\circ} \alpha \beta^{\circ}}(\omega)=\int_{-\infty}^{\infty}\left\langle\left(\alpha|K(t)| \alpha^{\prime}\right)\left(\alpha|K(t-\tau)| \beta^{\prime}\right)\right\rangle_{e} e^{-i \omega \tau} d \tau$.

However, since the two terms in these matrix elements have different selection rules and correspond to parts of the Hamiltonian assumed to be uncorrelated, they vanish. The resulting form of the off-diagonal matrix is simply

$$
\dot{\rho}_{\alpha \alpha^{\prime}}^{*}=R_{\alpha \alpha^{\prime} \alpha \alpha^{\prime}} \rho_{\alpha \alpha^{\prime}}^{*}
$$

with the solution

$$
\rho_{\alpha \alpha^{\prime}}^{*}(t)=e^{-\lambda \alpha \alpha^{\prime} t} \rho_{\alpha \alpha^{\prime}}^{*}(0),
$$

corresponding to (2.22) of I.

In the case of integral spins the structure of the diagonal part of I remains unchanged, but for the off-diagonal part the situation is different. In particular, spectral-density functions of the type (3), when either $\alpha$ or $\beta$ is zero, have the form

$J_{0-\beta 0 \beta}(\omega)=\int_{-\infty}^{\infty}\langle(0|K(t)|-\beta)(\beta|K(t+\tau)| 0)\rangle_{e} e^{-i \omega \tau} d \tau$,

which now is nonzero, since the two matrix elements involve the same selection rule. Thus, the relaxation matrix elements of the form $R_{\alpha \alpha^{\prime}-\alpha_{-} \alpha^{\prime}}$, $R_{\alpha \alpha^{\prime}-\alpha \alpha^{\prime}}$, and $R_{\alpha \alpha^{\prime} \alpha-\alpha^{\prime}}$ are nonzero if either $\alpha$ or $\alpha^{\prime}$ is zero.

As an illustration, we show the equations corresponding to (4) for the off-diagonal components in the case $\alpha^{\prime}=0$. We obtain the two coupled equations

$$
\begin{gathered}
\rho_{\alpha 0}^{*}(t)=\left(1 / \hbar^{2}\right)\left[R_{\alpha 0 \alpha 0} \rho_{\alpha 0}^{*}(t)+R_{\alpha 0-\alpha 0} \rho_{-\alpha 0}^{*}(t)\right], \\
\rho_{-\alpha 0}^{*}(t)=\left(1 / \hbar^{2}\right)\left[R_{-\alpha 0-\alpha 0} \rho_{-\alpha 0}^{*}(t)+R_{-\alpha 0 \alpha 0} \rho_{\alpha 0}^{*}(t)\right] .
\end{gathered}
$$

These can be rewritten as

$$
\begin{aligned}
\hbar^{2} \rho_{\alpha 0}^{*}(t)-R_{\alpha 0 \alpha 0} \rho_{\alpha 0}^{*}(t) & =R_{\alpha 0-\alpha 0} \rho_{-\alpha 0}^{*}(t), \\
\hbar^{2} \rho_{\alpha 0}^{*}(t)-R_{-\alpha 0-\alpha 0} \rho_{-\alpha 0}^{*}(t) & =R_{-\alpha 0 \alpha 0} \rho_{\alpha 0}^{*}(t) .
\end{aligned}
$$

We can try for $\rho_{\alpha \alpha^{\prime}}^{*}$ a solution of the type

$$
\rho^{*}(t)_{ \pm\left(\alpha \alpha^{\prime}\right)}=\rho^{*}(0)_{ \pm\left(\alpha \alpha^{\prime}\right)} e^{-\lambda \alpha \alpha^{\prime \prime}} \text {. }
$$

Substituting Eq. (9) into Eq. (8) one obtains

$$
\begin{aligned}
\left(\lambda \hbar^{2}-R_{\alpha 0 \alpha 0}\right) \rho_{\alpha 0}^{*}(0) & =R_{\alpha 0-\alpha 0} \rho_{-\alpha 0}^{*}(0), \\
\left(\lambda \hbar^{2}-R_{-\alpha 0-\alpha 0}\right) \rho_{-\alpha 0}^{*}(0) & =R_{-\alpha 0 \alpha 0} \rho_{\alpha 0}^{*}(0) .
\end{aligned}
$$

However, examination of the definitions shows that for quadrupole interactions

$$
\begin{aligned}
& R_{\alpha 0 \alpha 0}=R_{-\alpha 0-\alpha 0}=a, \\
& R_{\alpha 0-\alpha 0}=R_{-\alpha 0 \alpha 0}=b,
\end{aligned}
$$

which lead us to the eigenequation

$$
\left(\lambda \hbar^{2}-a\right)\left[\rho_{\alpha 0}^{*}(0) \pm \rho_{-\alpha 0}^{*}(0)\right]=b\left[\rho_{-\alpha 0}^{*}(0) \pm \rho_{\alpha 0}^{*}(0)\right] .
$$

Analyzing the sum and the difference in the brackets of Eq. (12), using the definitions of $\rho_{\alpha 0}^{*}(0)$ given by Eq. (2.3) in I, we see that the possible eigenvalues for $\lambda$ are

$$
\begin{aligned}
& \lambda^{+}=(b+a) / \hbar^{2}, \\
& \lambda^{-}=(b-a) / \hbar^{2},
\end{aligned}
$$

which give for the components of the density matrix

$$
\begin{aligned}
& \rho_{\alpha 0}^{*}(t)=\frac{1}{2}\left[e^{-\lambda^{+} t}+e^{-\lambda^{-t} t}\right]\left[\rho_{\alpha 0}^{*}(0)-\rho_{-\alpha 0}^{*}(0)\right], \\
& \rho_{-\alpha 0}^{*}(t)=\frac{1}{2}\left[e^{-\lambda^{+} t}+e^{-\lambda^{-} t}\right]\left[\rho_{-\alpha 0}^{*}(0)-\rho_{\alpha 0}^{*}(0)\right],
\end{aligned}
$$

whereas for those terms not involving either $\alpha$ or $\alpha^{\prime}$ equal zero the solution remains that of Eq. (5).

The perturbation factors in any particular case could be calculated using Eqs. (2.28) and (3.1) of I. We see that for the transition to ground state an additional sum of exponentials will appear in the perturbation factors derived for the odd- $A$ nuclei case.
*Work partially supported by BNDE (Brasil), IAEA and U. S. Air Force Office of Scientific Research, Grant No. AF-AFOSR-1280-67.

†Senior Research Fellow, Conselho Nacional de Pesquisas (Brasil).

\$isiting Professor, Regional Program of Scientific and Technological Development, OAS.
${ }^{1}$ P. da R. Andrade, J. D. Rogers, and A. Vasquez, Phys. Rev. 188, 571 (1969).

${ }^{2}$ J. C. Glass and J. K. Kliwer, Nucl. Phys. A115, 234 (1968).

${ }^{3}$ A. Abragam, Principles of Nuclear Magnetism (Oxford U. P., Oxford, England, 1961), Chap. X. 